CARDIOVASCULAR MEDICINE

\title{
Hyperuricaemia does not impair cardiovascular function in healthy adults
}

\author{
W S Waring, S H Adwani, O Breukels, D J Webb, S R J Maxwell
}

Heart 2004;90:155-159. doi: 10.1136/hrt.2003.016121

See end of article for authors' affiliations

.....................

Correspondence to: Dr W S Waring, Clinical Pharmacology Unit, The University of Edinburgh, Western General Hospital, Crewe Road, Edinburgh, UK; s.waring@ed.ac.uk

Accepted 20 June 2003
Objective: To investigate the possibility that uric acid (UA) can impair endothelial function, an important surrogate for atherosclerosis.

Design: UA was administered locally or systemically to healthy adult men and women in a series of randomised placebo controlled studies. This temporarily raised serum UA concentrations, so that the potential effects of hyperuricaemia on mechanisms of cardiovascular disease could be studied.

Main outcome measures: The effects of UA administration on basal blood flow and responses to locally administered acetylcholine, sodium nitroprusside, and $L-N^{G}$-monomethylarginine were studied in the forearm vascular bed with venous occlusion plethysmography. The effects of hyperuricaemia on systemic vascular resistance, large artery compliance, and baroreflex sensitivity were examined by validated noninvasive techniques.

Results: UA administration caused a twofold increase in serum concentrations. However, there were no acute effects on haemodynamic variables, basal forearm blood flow, or nitric oxide dependent endothelial function.

Conclusion: Unlike other risk factors associated with endothelial dysfunction, acute exposure to high concentrations of UA does not impair cardiovascular function in healthy men. These findings do not support a causal link between hyperuricaemia and atherosclerosis.
$\mathrm{U}$ ric acid (UA) is formed as a natural product of purine metabolism. Humans are exposed to comparatively high serum and tissue concentrations because of the lack of urate oxidase, an enzyme that is responsible for further metabolism of UA in virtually all other species. ${ }^{1} \mathrm{~A}$ physiological role of UA has been suggested on the basis of its potent antioxidant properties, ${ }^{2}$ although this potential benefit may be gained at the cost of an increased risk of joint and kidney disease. Epidemiological studies have identified a strong relation between hyperuricaemia and subsequent cardiovascular disease risk in unselected populations ${ }^{3-5}$ and high risk groups, including patients with hypertension, diabetes mellitus, and chronic cardiac failure. ${ }^{6-8}$ This relation has been attributed to associations between UA and potential confounding risk factors. ${ }^{3}$ However, several epidemiological studies have shown that the predictive power of hyperuricaemia persists, even after considering these risk factors. ${ }^{45}$ The distinction between UA as a coincidental or causal risk factor is important because, if UA is causal, treatment to lower serum UA concentrations may potentially reduce cardiovascular disease risk. Epidemiological studies are unlikely to resolve this issue. Given the high prevalence of cardiovascular disease, there is a pressing need to identify additional treatable risk factors. The role of UA in the cardiovascular system is poorly understood and potential mechanisms by which it may promote atherogenesis have received little attention. ${ }^{9}$

In health, the endothelium plays a pivotal part in regulating the cardiovascular system through the release of nitric oxide, which causes vasodilatation, inhibits platelet aggregation, and reduces local vascular inflammation. ${ }^{10}$ Impaired blood flow responses to endothelium dependent vasodilators are a characteristic finding in patients with any one of several major cardiovascular risk factors and are thought to be an important early step in the development of atherosclerosis. ${ }^{11} 12$ Disruption of endothelium dependent nitric oxide bioavailability also manifests as reduced large artery compliance ${ }^{13}$ and impaired baroreflex sensitivity (BRS). ${ }^{14}$ An inverse relation between serum UA concentration and nitric oxide activity has been identified, ${ }^{15}$ and it is therefore possible that UA directly influences endothelial function, either by causing endothelial dysfunction or, as an antioxidant, by protecting against the impairment of endothelial function. One study has reported a lower cardiovascular mortality among patients with heart failure treated with allopurinol and the authors attributed this to lowering of serum $\mathrm{UA}^{16}$ although there may be other explanations. The feasibility of administering UA in solution, for clinical studies, has recently been established. ${ }^{17}$ The purpose of the present research was to determine whether raised UA concentrations disrupt nitric oxide mediated vascular function, in the same manner as established cardiovascular risk factors. Therefore, the cardiovascular effects of acute hyperuricaemia were studied in healthy volunteers with no identifiable major cardiovascular risk factors.

\section{METHODS}

\section{Study group}

The local research ethics committee granted approval for the studies and written informed consent was obtained from each participant. The investigation conforms to the principles outlined in the Declaration of Helsinki. Participants were recruited from a community database of healthy volunteers held at the Clinical Research Centre of the University of Edinburgh. Studies were performed in the morning, after an overnight fast, in a quiet room maintained at $24-26^{\circ} \mathrm{C}$. Inclusion criteria were male or female sex and age 18 to 45

Abbreviations: ACh, acetylcholine; BP, blood pressure; BRS, baroreflex sensitivity; L-NMMA, L- $N^{G}$-monomethylarginine; Pl, pulse interval; SNP, sodium nitroprusside; UA, uric acid 
years. Exclusion criteria were increased blood pressure (BP) (> 160/100 mm Hg), clinical history of joint, kidney or cardiovascular disease, any medication being taken, regular tobacco use, serum creatinine $>110 \mu \mathrm{mol} / \mathrm{l}$, or serum UA $>400 \mu \mathrm{mol} / \mathrm{l}$.

\section{Intra-arterial drug administration}

The brachial artery of the non-dominant arm was cannulated with a 27 standard wire gauge steel needle (Cooper's Needle Works Ltd, Birmingham, UK) under local anaesthesia by an aseptic technique. Vasoactive drugs were administered through a 16 gauge epidural catheter (Portex Ltd, Kent, UK) connected to an IVAC P1000 syringe pump (Alaris Medical Ltd, Hampshire, UK). ${ }^{11}$ Saline was infused for 30 minutes at the start of the study and for 20 minutes between drugs to establish baseline blood flow. Vasoactive drugs were infused for six minutes at each dose and the rate of infusion was kept constant at $1 \mathrm{ml} / \mathrm{min}$ throughout.

\section{Measurement of forearm blood flow}

Blood flow was measured in both forearms by venous occlusion plethysmography, as previously described..$^{11} 18$ Measurements were taken during the last three minutes of each six minute infusion. The last five recordings were averaged to determine flow in each arm. Responses were expressed as percentage change from baseline, where the ratio of blood flow in the infused to that in the non-infused limbs was considered, to account for any systemic effects. ${ }^{11}$

\section{Systemic vascular resistance}

$\mathrm{BP}$ was recorded in the dominant arm with a validated oscillometric device (HEM-705CP, Omron, Kyoto, Japan). ${ }^{19}$ Cardiac index was assessed by transthoracic bioimpedance (NCCOM3-R7, BoMed, Irvine, California, USA). ${ }^{20}$ The systemic vascular resistance index was calculated as the mean arterial pressure divided by the cardiac index.

\section{Pulse wave analysis}

The dominant radial artery pulse waveform was assessed by applanation tonometry (SPC-301 micromanometer, Millar Instruments, Houston, Texas, USA). A corresponding aortic pressure waveform was generated by pulse wave analysis software (SphygmoCor, PWV, Sydney, Australia). ${ }^{21}$ The augmentation index is a validated measure of large artery stiffness, calculated as the difference between the first and second central systolic BP peaks, expressed as a percentage of pulse pressure. ${ }^{22}$

\section{Spontaneous BRS}

Study participants rested supine. Systolic BP was recorded continuously by a Portapres system (TNO, Amsterdam, the Netherlands) and an ECG was measured simultaneously. ${ }^{23} 24$ Signals from both devices were recorded over 15 minutes and analysed off line by Chart HRV software (ADInstruments, Hastings, UK). BRS was determined by two independent methods. Parallel increases or decreases in BP and pulse interval (PI) are thought to represent spontaneous baroreflex activity. ${ }^{25}$ Sequences of parallel increases or decreases over two or more consecutive beats were analysed and the resulting slope $(\triangle \mathrm{PI} / \triangle \mathrm{BP})$ was used to represent $\mathrm{BRS}$ by sequence analysis. ${ }^{25}$ Fast Fourier transformation of BP and PI data gave the total spectral power of the variability of each and the formula ( power $_{\mathrm{BP}} /$ power $\left._{\mathrm{PI}}\right)^{1 / 2}$ gave spontaneous BRS by spectral analysis. ${ }^{26}$

\section{Measurement of biochemical variables}

Blood was collected in gel tubes (Sarstedt Ltd, Leicester, UK), allowed to clot, and centrifuged at $1000 \mathrm{~g}$ for 10 minutes. Serum was separated and UA concentration was determined by a colorimetric dry slide method (Vitros, Ortho-Clinical Diagnostics, Amersham, UK).

\section{Drugs and reagents}

UA and lithium carbonate (Ultrapure preparations, Sigma Chemical Company, Poole, UK) were reconstituted in a sterile dextrose solution (Baxter Healthcare, Norfolk, UK) and filtered $(0.22 \mu \mathrm{m}$ Millex, Millipore, Molsheim, France). The drugs used were acetylcholine (ACh; CIBAVisionOphthalmics, Southampton, UK), sodium nitroprusside (SNP; David Bull Laboratories, Warwick, UK), and $\mathrm{L}-N^{\mathrm{G}}$ monomethylarginine (L-NMMA; Calbiochem-Novobiochem, Nottingham, UK).

\section{Basal forearm blood flow}

Six healthy men aged 29 (4) years (SEM) were recruited to a two way randomised placebo controlled study. Participants underwent intra-arterial administration of saline for 20 minutes to establish baseline blood flow, followed by infusion of $0,0.5,1.0,2.0$, and $4.0 \mathrm{mg} / \mathrm{min} \mathrm{UA}$ in $4 \%$ dextrose $/ 0.1 \%$ lithium carbonate vehicle for six minutes at each dose and for 12 minutes at the maximum dose. Forearm blood flow was assessed at baseline and during each infusion.

\section{Local hyperuricaemia and endothelial function}

Ten healthy men were recruited to a two way randomised placebo controlled study. They underwent intra-arterial administration of saline for 30 minutes to establish baseline blood flow, followed by ACh 7.5, 15, and $30 \mathrm{mmol} / \mathrm{min}$, SNP 2,4 , and $8 \mathrm{mmol} / \mathrm{min}$, and L-NMMA 2 and $4 \mu \mathrm{mol} / \mathrm{min}$, where the order of infusion of ACh and SNP was randomised between subjects. Drug infusions were separated by saline for 20 minutes to allow restoration of basal blood flow. Drugs were infused for six minutes at each dose. ${ }^{11}$ UA $2.0 \mathrm{mg} / \mathrm{min}$ in $4 \%$ dextrose $/ 0.1 \%$ lithium carbonate vehicle or vehicle alone was co-infused locally. Effluent venous blood ( $5 \mathrm{ml}$ ) was collected from each forearm during infusion for UA measurement.

\section{Systemic hyperuricaemia and endothelial function}

Ten healthy men were recruited to a two way randomised placebo controlled study. An 18 standard gauge venous cannula was inserted into a suitable vein in each antecubital fossa under local anaesthetic. Participants underwent systemic administration of $1000 \mathrm{mg}$ UA in $4 \%$ dextrose/0.1\% lithium carbonate vehicle or vehicle alone over one hour through the cannula in the non-dominant forearm. Immediately after infusion, endothelial function was studied as described above. Venous blood $(5 \mathrm{ml})$ was drawn from the non-infused forearm cannula at baseline, immediately after infusion, and one hour after infusion for UA measurement.

\section{Systemic haemodynamic variables}

Eight healthy men were recruited to a three way randomised placebo controlled study. An 18 standard gauge venous cannula was inserted into a suitable vein in each antecubital fossa under local anaesthetic. Portapres finger cuff and BoMed electrodes were applied. Participants rested supine for 30 minutes, then underwent systemic administration of $1000 \mathrm{mg}$ UA in $500 \mathrm{ml}$ 4\% dextrose/0.1\% lithium carbonate vehicle, vehicle alone, or $0.9 \%$ saline over one hour through the non-dominant forearm cannula. Electrocardiogram and Portapres signals were recorded for BRS determination at baseline and at the end of the infusion. BP, cardiac index, and pulse wave analysis (PWA) were measured at baseline and at 15 minute intervals. Venous blood $(5 \mathrm{ml})$ was drawn through the non-infused forearm cannula at baseline and immediately after infusion for UA measurement. 


\section{Data analysis and statistics}

Numbers of study participants were determined to give at least $80 \%$ power to detect a $10 \%$ difference in the primary outcome variables (forearm blood flow response to ACh, augmentation index, and BRS). Responses were compared by two way analysis of variance and paired Student's $t$ tests, where appropriate. Significance was accepted at the 5\% level in all cases. All values are reported as mean (SEM).

\section{RESULTS}

Table 1 shows baseline characteristics of the study participants.

\section{Basal blood flow}

Local administration of neither vehicle nor UA had any effect on basal forearm blood flow (fig 1). Local UA and vehicle administrations caused systemic UA concentrations to rise by 62 (13) and -4 (3) $\mu \mathrm{mol} / \mathrm{l}$, respectively ( $<<0.001$ ); $69 \mathrm{mg}$ (about $410 \mu \mathrm{mol}$ ) UA was administered to each subject and the mean volume of distribution was calculated to be 22.6 (2.0) 1 .

\section{Local hyperuricaemia and endothelial function}

Venous effluent UA concentrations in the infused and noninfused forearms were 384 (7) and 280 (1) $\mu \mathrm{mol} / \mathrm{l}$, respectively, during UA administration $(\mathrm{p}<0.001)$ and 290 (4) and 283 (1) $\mu \mathrm{mol} / \mathrm{l}$, respectively, during vehicle administration. This was an increase of $33(3) \%$ and of $-1(0) \%$ during $\mathrm{UA}$ and vehicle administration, respectively $(\mathrm{p}<0.001)$. Despite this, responses to ACh, SNP, and L-NMMA were unaltered in the forearm vascular bed (fig 2).

\section{Systemic hyperuricaemia and endothelial function}

Serum UA concentrations before, immediately after, and one hour after infusion were 227 (8), 534 (18), and 452 (11) $\mu \mathrm{mol} / \mathrm{l}$, respectively, for UA administration and 224 (27), 220 (27), and 217 (27) $\mu \mathrm{mol} / \mathrm{l}$, respectively, for vehicle administration. These were increases of 145 (19)\% and -4 ( 1$) \%$ for UA and vehicle, respectively $(p<0.001)$. Forearm blood flow responses to ACh, SNP, and L-NMMA infusion were not altered by systemic hyperuricaemia (fig 3).

\section{Systemic haemodynamic variables}

Baseline versus post-infusion serum UA concentrations were 336 (12) $v 350$ (1) $\mu \mathrm{mol} / \mathrm{l}, 370$ (17) $v 361$ (17) $\mu \mathrm{mol} / \mathrm{l}$, and 370 (16) $v 627(23) \mu \mathrm{mol} / \mathrm{l}(\mathrm{p}<0.001)$ for saline, vehicle, and UA administration, representing increases from baseline of $-4(1) \%,-2(1) \%$, and $79(7) \%$, respectively $(p<0.001)$. Augmentation index, central systolic BP, BRS by sequence analysis, and BRS by spectral analysis were not altered by systemic hyperuricaemia (table 2). The systemic vascular

Table 1 Study subjects baseline characteristics

\begin{tabular}{llll}
\hline & $\begin{array}{l}\text { Local UA } \\
\text { and } \\
\text { endothelial } \\
\text { function }\end{array}$ & $\begin{array}{l}\text { Systemic } \\
\text { UA and } \\
\text { endothelial } \\
\text { function }\end{array}$ & $\begin{array}{l}\text { Systemic } \\
\text { UA and } \\
\text { haemodynamic } \\
\text { variables }\end{array}$ \\
\hline Number $/ \mathrm{men}$ & $10 / 10$ & $10 / 6$ & $8 / 8$ \\
Age (years) & $23(1)$ & $24(1)$ & $30(4)$ \\
Systolic BP $(\mathrm{mm} \mathrm{Hg})$ & $110(4)$ & $107(6)$ & $108(3)$ \\
Diastolic BP $(\mathrm{mm} \mathrm{Hg})$ & $68(5)$ & $71(4)$ & $72(3)$ \\
Heart rate $(\mathrm{beats} / \mathrm{min})$ & $59(2)$ & $58(3)$ & $60(2)$ \\
Body mass index $\left(\mathrm{kg} / \mathrm{m}^{2}\right)$ & $23(1)$ & $22(1)$ & $23(0)$ \\
Creatinine $(\mu \mathrm{mol} / /)$ & $84(4)$ & $84(6)$ & $86(3)$ \\
Glucose $(\mathrm{mmol} / \mathrm{l})$ & $4.6(0.2)$ & $4.8(0.1)$ & $4.7(0.3)$ \\
Cholesterol $(\mathrm{mmol} / \mathrm{l})$ & $4.1(0.2)$ & $4.1(0.2)$ & $4.8(0.2)$ \\
Uric acid $(\mu \mathrm{mol} / \mathrm{l})$ & $258(13)$ & $252(14)$ & $366(12)$ \\
\hline
\end{tabular}

Data are mean (SEM).

$\mathrm{BP}$, blood pressure; UA, uric acid.

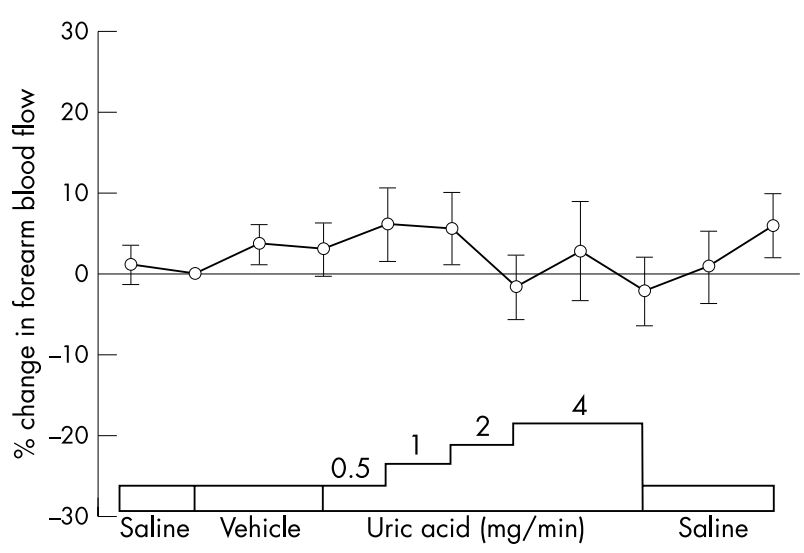

Figure 1 Pilot study. Forearm blood flow, as the ratio of the infused to the non-infused forearm, expressed as percentage change from baseline during local administration of uric acid (UA; $0,0.5,1.0,2.0$, and $4.0 \mathrm{mg} / \mathrm{min}$ ) in the vehicle $(\mathrm{n}=6)$.

resistance index increased during all infusions, with a nonsignificant trend towards lower increases during UA infusion (table 2).

\section{DISCUSSION}

The importance of high serum UA concentration as a marker of increased cardiovascular risk has been recognised for more than 50 years. ${ }^{27}$ However, no biologically plausible causal link to atherosclerosis has been shown in vivo. In the current study, UA administration had no effect on basal forearm blood flow or response to L-NMMA, indicating that short lived hyperuricaemia does not have a direct impact on resting vascular tone or basal nitric oxide release. Lack of effect on endothelium dependent and endothelium independent vasodilator responses suggests that high UA concentrations do not affect vascular smooth muscle nitrate responsiveness or stimulate nitric oxide release in health.

There is a strong association between disease states characterised by loss of vascular nitric oxide activity and high serum UA concentrations. The present findings indicate that acute hyperuricaemia does not directly influence constitutive, or stimulated, nitric oxide liberation from the vascular endothelium. A previous study has shown that enhancing vascular nitric oxide bioavailability by L-arginine supplementation causes a reduction in circulating UA concentrations. ${ }^{15}$ Therefore, UA may be responsive to vascular nitric oxide activity, consistent with a non-causal association between endothelial dysfunction and increased serum UA concentrations. Furthermore, UA is an important intracellular free radical scavenger during metabolic stress, ${ }^{28}$ for example, in vascular smooth muscle and endothelial cells, ${ }^{29}$ and circulating concentrations are thought to be responsive to the local redox state. ${ }^{31}$ Therefore, it is possible that increased UA concentrations are a compensatory response, in view of the antioxidant properties of UA.

Hyperuricaemia coexists with impaired large artery compliance in several disease states characterised by reduced vascular nitric oxide bioavailability. UA had no impact on the large conduit vessels that determine pulse waveform conduction, suggesting that high concentrations are not causally linked to increased vascular stiffness. BRS, determined by two discrete methods, ${ }^{25}{ }^{26}$ was unaffected by UA, consistent with the lack of effect on constitutive nitric oxide activity and large artery compliance, which are important vessel wall properties that can influence BRS. The lack of effect on BRS also indicates that baroreceptor function, and indeed cardiac sensitivity to autonomic outflow, was not affected by local 

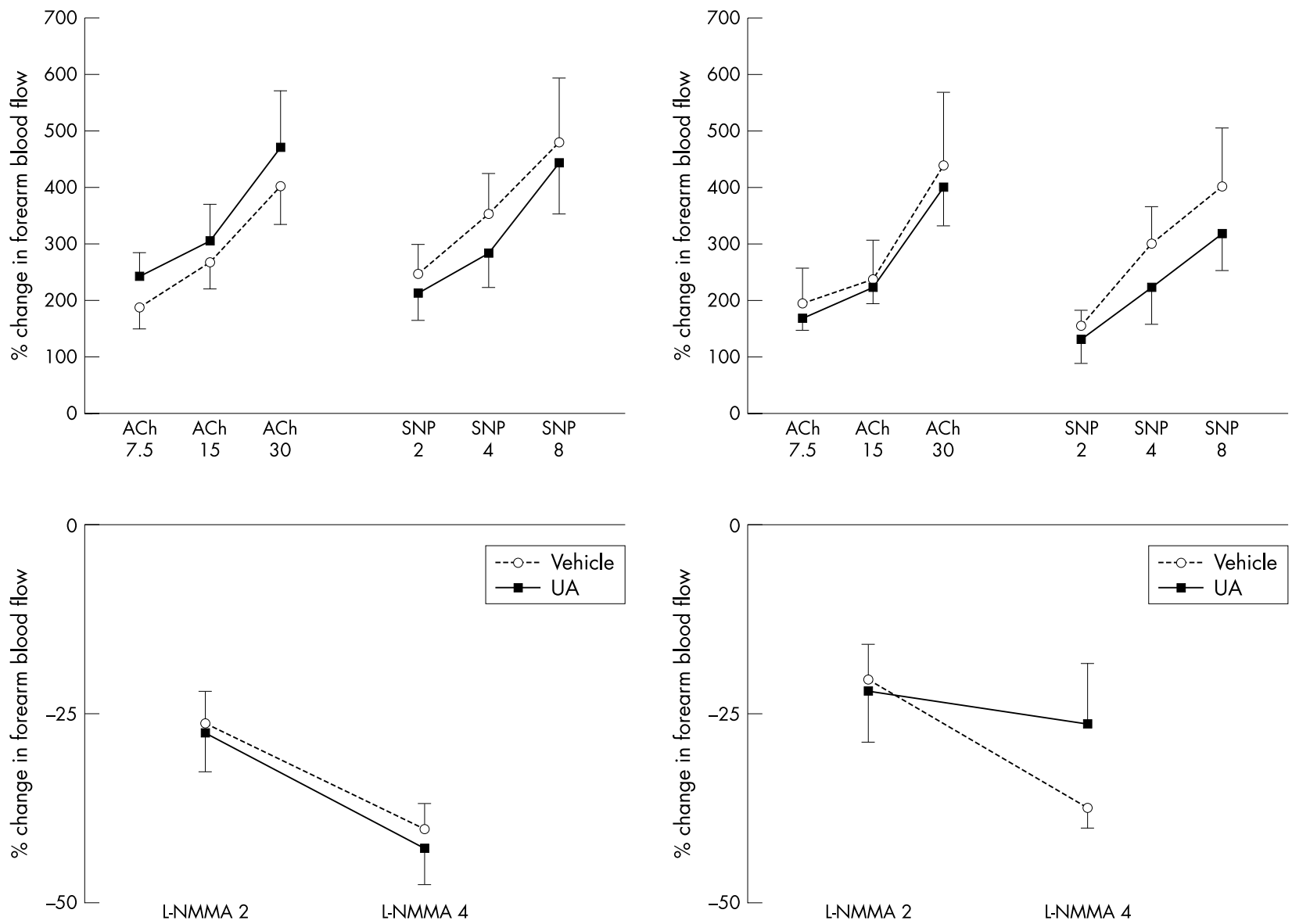

Figure 2 Forearm blood flow responses to acetylcholine (ACh) 7.5, 15, and $30 \mu \mathrm{g} / \mathrm{min}$, nitroprusside (SNP) 2,4 , and $8 \mu \mathrm{g} / \mathrm{min}$, and $\mathrm{L}-\mathrm{N}^{G}$. monomethylarginine (L-NMMA) 2 and $4 \mu \mathrm{mol} / \mathrm{min}$, as a ratio of the infused to the non-infused forearm and expressed as percentage change from baseline during local co-administration of $2 \mathrm{mg} / \mathrm{min}$ UA in vehicle or of vehicle alone $(p=0.74,0.47$, and 0.87 , respectively; $n=10)$.

increases in UA concentration, irrespective of vessel wall conditions.

There are several important limitations of these findings. This series of acute, mechanistic studies did not address the effects of chronic exposure to increased serum UA concentrations, which may be a more important determinant of future cardiovascular risk. However, it is less feasible to examine chronic hyperuricaemia in a controlled manner because of the potential risks of joint and kidney disease. A

Figure 3 Forearm blood flow responses to $A C h 7.5,15$, and $30 \mu \mathrm{g} /$ $\mathrm{min}, \mathrm{SNP} 2,4$, and $8 \mu \mathrm{g} / \mathrm{min}$, and L-NMMA 2 and $4 \mu \mathrm{mol} / \mathrm{min}$, as a ratio of the infused to the non-infused forearm and expressed as percentage change from baseline after systemic administration of $1000 \mathrm{mg}$ UA in vehicle or of vehicle alone $(p=0.87,0.65$, and 0.38 , respectively; $\mathrm{n}=10$ ).

further potential limitation is that the current studies addressed the effects of an acute increase of UA in a young, healthy population, free from major cardiovascular risk factors. Nonetheless, if UA were an independent causal risk factor for atherosclerosis, then its presence should be expected to impair endothelial function. Other atherosclerotic risk factors cause acute impairment of endothelial function in young, healthy people-for example, increased homocysteine concentrations after methionine administration ${ }^{32}$ or ingestion

Table 2 Systemic haemodynamic variables before and after systemic administration of $1000 \mathrm{mg}$ UA in vehicle, vehicle alone, or saline $(\mathrm{n}=8)$

\begin{tabular}{|c|c|c|c|c|c|c|}
\hline & \multicolumn{2}{|l|}{ Saline } & \multicolumn{2}{|l|}{ Vehicle } & \multicolumn{2}{|l|}{ UA } \\
\hline & Baseline & Postinfusion & Baseline & Postinfusion & Baseline & Postinfusion \\
\hline $\mathrm{SBP}(\mathrm{mm} \mathrm{Hg})$ & $96(3)$ & $97(3)$ & $93(2)$ & $97(5)$ & $96(4)$ & $100(4)$ \\
\hline $\mathrm{DBP}(\mathrm{mm} \mathrm{Hg})$ & $61(2)$ & $65(2)$ & $63(3)$ & $68(3)$ & $62(4)$ & $71(3)$ \\
\hline HR (beats/min) & $55(3)$ & $53(3)$ & $57(4)$ & $58(3)$ & $58(3)$ & $59(2)$ \\
\hline Alx $(\%)$ & $-6.7(6.0)$ & $-3.1(6.1)$ & $-2.6(6.0)$ & $-1.1(6.1)$ & $-3.6(5.1)$ & $-6.1(6.2)$ \\
\hline $\mathrm{Cl}\left(\mathrm{l} / \mathrm{min} / \mathrm{m}^{2}\right)$ & $3.3(0.2)$ & $3.0(0.2)$ & $3.3(0.4)$ & $3.3(0.3)$ & $3.1(0.2)$ & $3.2(0.3)$ \\
\hline SVRI (au) & $12.0(1.0)$ & $13.7(0.9)$ & $10.2(1.5)$ & $11.7(2.0)$ & $12.3(1.0)$ & $13.0(1.0)$ \\
\hline $\mathrm{BRS}_{\mathrm{seg}}(\mathrm{ms} / \mathrm{mm} \mathrm{Hg})$ & $22.3(4.7)$ & $21.2(4.0)$ & $19.7(4.9)$ & $22.8(5.0)$ & $21.8(4.4)$ & $20.3(4.4)$ \\
\hline $\mathrm{BRS}_{\text {spec }}(\mathrm{ms} / \mathrm{mm} \mathrm{Hg})$ & $24.3(5.8)$ & $19.4(3.4)$ & $22.2(6.6)$ & $26.8(6.4)$ & $23.4(5.0)$ & $21.7(5.9)$ \\
\hline
\end{tabular}

Data are mean (SEM).

$\mathrm{Alx}$, augmentation index; au, arbitrary units; $\mathrm{BRS}_{\text {seq, }}$ baroreflex sensitivity by sequence analysis; $\mathrm{BRS}_{\text {spec, }}$ baroreflex sensitivity by spectral analysis; $\mathrm{Cl}$, cardiac index; DBP, diastolic blood pressure; HR, heart rate; SBP, systolic blood pressure; SVRI, systemic vascular resistance index 
of a fatty meal..$^{33}$ Additionally, amelioration of established risk factors allows rapid restoration of endothelial function, including correction of hypertension, hypercholesterolaemia, or hyperhomocysteinaemia.

A further limitation is that the effects of raising UA concentrations on endothelial function were studied only in healthy men. Previous studies have shown that the relation between hyperuricaemia and increased cardiovascular risk is more apparent in women, in both unselected populations ${ }^{34}$ and those with established coronary artery disease. ${ }^{35}$ Additional research is required to investigate the effects of raised serum UA concentrations on endothelial function in women. Furthermore, the effects of raised serum UA concentrations merit further investigation in men and women with established cardiovascular risk factors.

The effects of raising UA concentrations may be modest because of the comparatively high background concentrations to which humans, as a species, are ordinarily exposed. Further work is required to establish the cardiovascular effects of lowering serum UA concentrations. Xanthine oxidase inhibitors-for example, allopurinol-cause a modest reduction in circulating UA concentrations. Xanthine oxidase activity produces hydrogen peroxide, an important source of free radicals in vivo, ${ }^{36}$ which confounds the relation between xanthine oxidase inhibitors and UA lowering. Methods that lower UA directly should allow more useful interpretation of any cardiovascular effects-for example, using urate oxidase, which causes rapid and substantial reductions in circulating UA concentrations. ${ }^{37}$

In summary, high serum UA concentrations, at least in the acute setting, do not impair cardiovascular function in healthy men. These findings do not support a causal role for UA in the development of atherosclerosis.

\section{ACKNOWLEDGEMENTS}

This work was funded, in part, by an endowment grant from the Faculty of Medicine of the University of Edinburgh (E80870). Dr W S Waring was supported by a two year Bristol-Myers Squibb Cardiovascular Research Fellowship and Professor DJ Webb was supported by a Research Leave Fellowship from the Wellcome Trust (WT 0526330) at the time the research was done.

\section{Authors' affiliations}

W S Waring, S H Adwani, D J Webb, S R J Maxwell, Centre for Cardiovascular Science, The University of Edinburgh, Edinburgh, UK O Breukels, Department of Pharmacy, Utrecht University, Utrecht, the Netherlands

\section{REFERENCES}

1 Oda M, Satta Y, Takenaka O, et al. Loss of urate oxidase activity in hominoids and its evolutionary implications. Mol Biol Evol 2002;19:640-53.

2 Ames BN, Cathcart R, Schwiers E, et al. Uric acid provides an antioxidant defense in humans against oxidant- and radical-caused aging and cancer: a hypothesis. Proc Natl Acad Sci U S A 1981;78:6858-62.

3 Brand FN, McGee DL, Kannel WB, et al. Hyperuricemia as a risk factor of coronary heart disease: the Framingham study. Am J Epidemiol $1985 ; 121: 11-8$

4 Levine W, Dyer AR, Shekelle RB, et al. Serum uric acid and 11.5 year mortality of middle-aged women findings of the Chicago Heart Association detection project in industry. J Clin Epidemiol 1989:42:257-67.

5 Freedman DS, Williamson DF, Gunter EW, et al. Relation of serum uric acid to mortality and ischemic heart disease. The NHANES I epidemiologic follow-up study. Am J Epidemiol 1995;141:637-44.

6 Alderman $\mathrm{MH}$, Cohen $\mathrm{H}$, Madhavan S, et al. Serum uric acid and cardiovascular events in successfully treated hypertensive patients. Hypertension 1999;34:144-50.

7 Staessen J, for members of the European Working Party on High Blood Pressure in the Elderly. The determinants and prognostic significance of serum uric acid in elderly patients of the European working party on high blood pressure in the elderly trial. Am J Med 1991;90(suppl 3a):50-4S.
8 Lehto S, Niskanen L, Ronnemaa T, et al. Serum uric acid is a strong predictor of stroke in patients with non-insulin-dependent diabetes mellitus. Stroke 1998;29:635-9.

9 Waring WS, Webb DJ, Maxwell SRJ. Uric acid as a risk factor for cardiovascular disease. Q J Med 2000;93:707-13.

10 Vallance $\mathbf{P}$. Nitric oxide in the human cardiovascular system. $\mathrm{Br} J \mathrm{Clin}$ Pharmacol 1998;45:433-9.

11 Wilkinson IB, Webb DJ. Venous occlusion plethysmography in cardiovascular research: methodology and clinical applications. Br J Clin Pharmacol 2001;52:631-46.

12 Heitzer T, Just H, Munzel T. Antioxidant vitamin C improves endothelial dysfunction in chronic smokers. Circulation 1996;94:6-9.

13 Smulyan H, Asmar RG, Rudnicki A, et al. Comparative effects of aging in men and women on the properties of the arterial tree. J Am Coll Cardiol 2001;37:1374-80.

14 Spieker LE, Corti R, Binggeli $\mathrm{C}$, et al. Baroreceptor dysfunction induced by nitric oxide synthase inhibition in humans. J Am Coll Cardiol 2000;36:213-8.

15 Maxwell AJ, Bruinsma KA. Uric acid is closely linked to vascular nitric oxide activity. Evidence for mechanism of association with cardiovascular disease. J Am Coll Cardiol 2001;38:1850-8.

16 Struthers AD, Donnan PT, Lindsay P, et al. Effect of allopurinol on mortality and hospitalisations in chronic heart failure: a retrospective cohort study. Heart 2002;87:229-34.

17 Waring WS, Webb DJ, Maxwell SR. Systemic uric acid administration increases serum antioxidant capacity in healthy volunteers. J Cardiovasc Pharmacol 2001;38:365-71.

18 Witherow FN, Helmy A, Webb DJ, et al. Bradykinin contributes to the vasodilator effects of chronic angiotensin-converting enzyme inhibition in patients with heart failure. Circulation 2001;104:2177-81.

19 O'Brien E, Mee F, Atkins N, et al. Evaluation of three devices for selfmeasurement of blood pressure according to the revised British Hypertension Society protocol: the Omron HEM-705CP, Philips HP5332, and Nissei DS175. Blood Press Monit 1996;1:55-61.

20 Thomas SH. Impedance cardiography using the Sramek-Bernstein method: accuracy and variability at rest and during exercise. $\mathrm{Br} J$ Clin Pharmacol 1992;34:467-76.

21 Wilkinson IB, Fuchs SA, Jansen IM, et al. Reproducibility of pulse wave velocity and augmentation index measured by the pulse wave analysis. $J$ Hypertens 1998;16:2079-84.

22 O'Rourke MF, Mancia G. Arterial stiffness. J Hypertens 1999;17:1-4.

23 Jones RD, Kornberg JP, Roulson CJ, et al. The Finapres 2300e finger cuff. The influence of cuff application on the accuracy of blood pressure measurement. Anaesthesia 1993;48:611-5.

24 Dawson SL, Panerai RB, Potter JF. Should one use electrocardiographic or Finapres-derived pulse intervals for calculation of cardiac baroreceptor sensitivity? Blood Press Monit 1998;3:315-20.

25 Parati G, Saul JP, Di Rienzo M, et al. Spectral analysis of blood pressure and heart rate variability in evaluating cardiovascular regulation. A critical appraisal. Hypertension 1995;25:1276-86.

26 lellamo F, Legramante JM, Raimondi G, et al. Evaluation of reproducibility of spontaneous baroreflex sensitivity at rest and during laboratory tests. J Hypertens 1996; 14:1099-104.

27 Gertler MM, Garn SM, Levy SA. Serum uric acid in relation to age and physique in health and in coronary heart disease. Ann Intern Med $1951 ; 34: 1421-31$

28 Hellsten Y, Tullson PC, Richter EA, et al. Oxidation of urate in human skeletal muscle during exercise. Free Radic Biol Med 1997;22:169-74.

29 Becker BF, Leipert B, Raschke $\mathrm{P}$, et al. Formation, release and scavenger function of uric acid derived from adenine nucleotides in heart and lung. In: Imai S, Nakazawa M, eds. Role of adenosine and adenine nucleotides in the biological system. Amsterdam: Elsevier Science Publishers, 1991:321-36.

30 Mathru M, Dries DJ, Barnes L, et al. Tourniquet-induced exsanguination in patients requiring lower limb surgery. An ischemia-reperfusion model of oxidant and antioxidant metabolism. Anesthesiology 1996;84:14-22.

31 Nowicki PT, Flavahan S, Hassanain H, et al. Redox signalling of the arteriolar myogenic response. Circ Res 2001;89:114-6.

32 Chambers JC, McGregor A, Jean-Marie J, et al. Demonstration of rapid onset vascular endothelial dysfunction after hyperhomocysteinemia: an effect reversible with vitamin C therapy. Circulation 1999;99:1156-60.

33 Williams MJ, Sutherland WH, McCormick MP, et al. Impaired endothelial function following a meal rich in used cooking fat. J Am Coll Cardiol 1999;33:1050-5.

34 Fang J, Alderman MH. Serum uric acid and cardiovascular mortality the NHANES I epidemiologic follow-up study, 1971-1992. National Health and Nutrition Examination Survey. J Am Med Assoc 2000;283:2404-10.

35 Aboa Eboule AC, De Smet P, Dramaix M, et al. [Relation between uricemia and total, cardiovascular and coronary mortality in both genders of nonselected sample of the Belgium population]. Rev Epidemiol Sante Publique 2001;49:531-9.

36 Rathaus M, Bernheim J. Oxygen species in the microvascular environment: regulation of vascular tone and the development of hypertension. Nephrol Dial Transplant 2002;17:216-21.

37 Pui CH. Urate oxidase in the prophylaxis or treatment of hyperuricemia: the United States experience. Semin Hematol 2001;38(suppl 10):13-21. 